\title{
Magnetic soliton: From two to three components with $\mathrm{SO}(3)$ symmetry
}

\author{
Xiao Chai $\odot,{ }^{1, *}$ Di Lao, ${ }^{1}$ Kazuya Fujimoto, ${ }^{2,3}$ and Chandra Raman ${ }^{1}$ \\ ${ }^{1}$ School of Physics, Georgia Institute of Technology, 837 State Street, Atlanta, Georgia 30332, USA \\ ${ }^{2}$ Institute for Advanced Research, Nagoya University, Nagoya 464-8601, Japan \\ ${ }^{3}$ Department of Applied Physics, Nagoya University, Nagoya 464-8603, Japan
}

(Received 26 October 2020; accepted 17 December 2020; published 6 January 2021)

\begin{abstract}
Recent theoretical and experimental research has explored magnetic solitons in binary Bose-Einstein condensates. Here we demonstrate that such solitons are part of an $\mathrm{SO}(3)$ soliton family when embedded within a full three-component spin-1 manifold with spin-rotational symmetry. To showcase this, we have experimentally created a type of domain wall magnetic soliton obtained by $90^{\circ}$ rotations, which consists of a boundary between easy-axis and easy-plane polar phases. Collisions between $\mathrm{SO}(3)$ solitons are investigated by numerically solving the Gross-Pitaevskii equations, which exhibit novel properties, including rotation and dissipation of soliton spin polarization.
\end{abstract}

DOI: 10.1103/PhysRevResearch.3.L012003

\section{INTRODUCTION}

Bose-Einstein condensates (BECs) of weakly interacting atoms offer a prominent platform for soliton studies [1-4]. In particular, they have become a versatile arena for research on vector solitons, where the rich internal hyperfine level structure of the atoms hosts a great deal of internal symmetry that allows for the observation of many types of solitons in the laboratory [5-8]. Indeed, symmetry has the potential to unify numerous vector soliton observations in the literature [9]. A powerful example of this is the connection between beating dark-dark solitons [10,11] and dark-bright solitons [6], which can be connected to one another via SU(2) rotations. Thus far such symmetry considerations have been applied only to solitons in the integrable Manakov limit [12], where the intraand interspecies interaction strengths are assumed to be equal. A far more diverse range of symmetries is possible in quantum gases with spin-dependent interaction, and their connection to vector solitons is a topic that has so far been unexplored.

In this work we uncover theoretically and provide experimental evidence for a diverse family of solitons in a spin-1 BEC with antiferromagnetic interactions. The recently observed two-component magnetic solitons [13,14] are shown to be but one member of this family. We numerically explore the consequences of $\mathrm{SO}(3)$ rotational symmetry for this family, as well as its breakdown in the presence of a finite quadratic Zeeman shift. $\mathrm{SO}(3)$ symmetry also creates entirely new possibilities for engineering collisions between solitons of different spin orientations, which we show numerically to

\footnotetext{
*xchai@gatech.edu

Published by the American Physical Society under the terms of the Creative Commons Attribution 4.0 International license. Further distribution of this work must maintain attribution to the author(s) and the published article's title, journal citation, and DOI.
}

have nontrivial spin rotation. To our knowledge these concepts have been applied only to Manakov systems, although a recent preprint has explored domain wall physics in the case of ferromagnetic interactions [15].

\section{FORMALISM}

Our work is situated in the mean-field description of a one-dimensional spin-1 BEC relevant to recent experiments $[13,14]$. It utilizes three coupled Gross-Pitaevskii equations (GPEs),

$$
\begin{aligned}
i \hbar \frac{\partial}{\partial t} \psi_{m}= & \left(-\frac{\hbar^{2}}{2 M} \frac{\partial^{2}}{\partial y^{2}}+\mathcal{V}\right) \psi_{m}+q m^{2} \psi_{m} \\
& +g_{0} n_{\mathrm{tot}} \psi_{m}+g_{2} \sum_{n=-1}^{1} \boldsymbol{F} \cdot(\hat{\boldsymbol{F}})_{m n} \psi_{n}
\end{aligned}
$$

where $\psi_{m}(y, t)$ is the order parameter with magnetic quantum number $m=-1,0,+1 . y$ and $t$ are space and time coordinates, respectively. $M$ is the atomic mass. $\mathcal{V}(y)$ and $q$ are the spin-independent potential and the quadratic Zeeman shift, respectively. $n_{\text {tot }}(y, t)=\sum_{m=-1}^{1}\left|\psi_{m}(y, t)\right|^{2}$ is the total density. $\boldsymbol{F}(y, t)=\sum_{m, n=-1}^{1} \psi_{m}^{*}(y, t)(\hat{\boldsymbol{F}})_{m n} \psi_{n}(y, t)$ is the spin density, and $\hat{\boldsymbol{F}}$ is the spin-1 matrix [16]. $g_{0}$ and $g_{2}$ are effective spin-independent and spin-dependent interaction coupling constants in a one-dimensional system, respectively. Here we consider only repulsive and antiferromagnetic interaction, namely, $g_{0}>g_{2}>0$.

In the absence of external fields, i.e., $\mathcal{V}(y)=0, q=0$, and assuming the total density is a constant $n$, the above equation has a magnetic soliton solution [13,14,17] when $g_{2} \ll g_{0}$. This condition can be fulfilled in a sodium BEC where $g_{2} / g_{0} \approx 0.036$ [18]. In a magnetic soliton solution, the $m=0$ component has no population, and the densities of 
$m= \pm 1$ are given by

$$
n_{ \pm 1}=\frac{n}{2}\left[1 \pm \sqrt{1-U^{2}} \operatorname{sech}\left(\sqrt{1-U^{2}} \zeta\right)\right]
$$

where $U=V / c_{s}$ is the soliton velocity $V$ normalized to that of spin waves, $c_{s}=\sqrt{n g_{2} / M} \cdot \zeta=(y-V t) / \xi_{s}$ is the moving coordinate, where the width of the soliton depends upon the spin healing length $\xi_{s}=\hbar / \sqrt{4 M n g_{2}}$. We can rewrite this solution in terms of the three-component spinor,

$$
\psi_{\mathrm{MS}}=\left(\begin{array}{c}
\sqrt{n_{+1}} e^{i \phi_{+1}} \\
0 \\
\sqrt{n_{-1}} e^{i \phi_{-1}}
\end{array}\right),
$$

where the wave functions have been written in terms of the amplitude and phase of each component. In Ref. [17], the phases $\phi_{A, B}=\phi_{+1} \mp \phi_{-1}$ are shown analytically to be

$$
\begin{aligned}
\cot \phi_{A} & =-\sinh \left(\zeta \sqrt{1-U^{2}}\right) / U, \\
\tan \left(\phi_{B}+C\right) & =-\sqrt{1-U^{2}} \tanh \left(\zeta \sqrt{1-U^{2}}\right) / U,
\end{aligned}
$$

where $C$ is chosen to ensure $\phi_{B}(\zeta=-\infty)=0$. It can be proven that such a solution is asymptotically valid in the limit $g_{2} \ll g_{0}[19]$, and we will assume its deviation from the true solution is negligible.

Under the Cartesian representation [20], a spin-1 spinor can be decomposed as $\left(\psi_{x}, \psi_{y}, \psi_{z}\right)^{t}=e^{i \phi}(\boldsymbol{u}+i \boldsymbol{v})$, where $\boldsymbol{u}$ and $\boldsymbol{v}$ are two real vectors obeying $|\boldsymbol{u}|^{2}+|\boldsymbol{v}|^{2}=n_{\mathrm{tot}}$, while $\phi$ is a global phase chosen to satisfy $\boldsymbol{u} \cdot \boldsymbol{v}=0$ and $|\boldsymbol{u}| \geqslant|\boldsymbol{v}|$. Thus, a spin-1 state can be fully characterized by $\boldsymbol{u}$ and $\boldsymbol{v}$. One can show that the spin density $\boldsymbol{F}=2 \boldsymbol{u} \times \boldsymbol{v}$ [21,22]; then $\boldsymbol{v}=-(\boldsymbol{u} \times \boldsymbol{F}) /\left(2|\boldsymbol{u}|^{2}\right)$, which indicates that the two vectors $\boldsymbol{F}$ and $\boldsymbol{u}$ can also determine a spin-1 state. In a spin-nematic state where $|\boldsymbol{F}|=0$, the vector $\boldsymbol{u}$ is called the director, whose direction plays the role of the order parameter [21]. In this Letter, we will generalize the definition of the director and call $\boldsymbol{u}$ the director in general cases when $|\boldsymbol{F}| \neq 0$. Figure 1(a) shows the spatially varying spin density $\boldsymbol{F}$ and director $\boldsymbol{u}$ for the magnetic soliton solution of Eq. (3) found by Qu et al. [17]. The spin density points along $\hat{z}$ and is localized near $y=0$, while the director rotates from the $+y$ direction to the $+x$ direction in that same region.

To extract the essential features of various solitons, we define the spin vector $\boldsymbol{S}$ as the normalized spin density at the center of the soliton

$$
\boldsymbol{S} \equiv \frac{\boldsymbol{F}(\zeta=0)}{n_{\mathrm{tot}}(\zeta=0)} .
$$

For the magnetic soliton solution (3), the spin vector is given by $\boldsymbol{S}=\sqrt{1-U^{2}}(0,0,1)^{t}$, whose amplitude is related to the soliton energy [17] as $\epsilon=n \hbar c_{s}|\boldsymbol{S}|$, while its direction characterizes the direction of polarization, which is the $+z$ direction in this case.

The spin- 1 system described by Eq. (1) conserves the total spin angular momentum and atom number in the absence of external fields, i.e., $q=0$. Thus, we have the symmetry group of the system [23],

$$
G=\mathrm{SO}(3)_{\hat{\boldsymbol{F}}} \times \mathrm{U}(1)_{\phi},
$$

where $\hat{\boldsymbol{F}}$ and $\phi$ denote spin and gauge degrees of freedom. Exploiting the $\mathrm{SO}(3)$ rotational symmetry in the spin degree of (a)

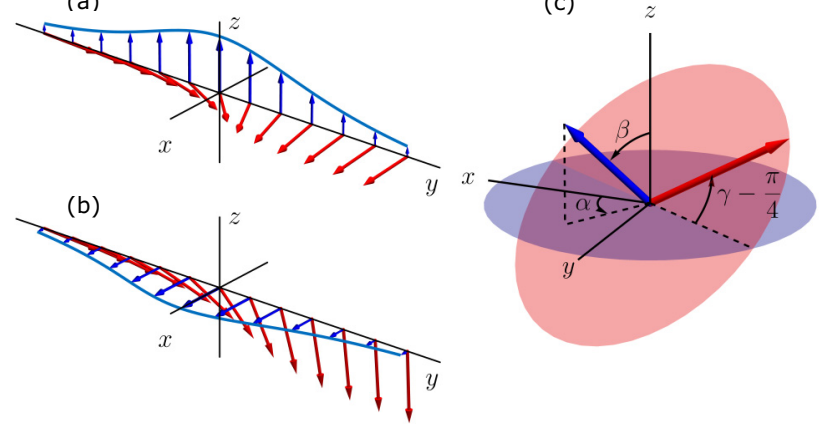

(d) $\alpha=0$

(e) $\alpha=0$

(f) $\alpha=0$

(g) $\alpha=3 \pi / 2$

$\beta=4 \pi / 3$

$\gamma=0$
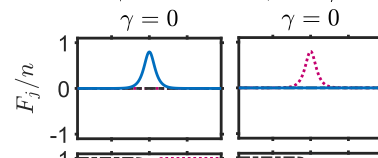

$\gamma=\pi / 4$
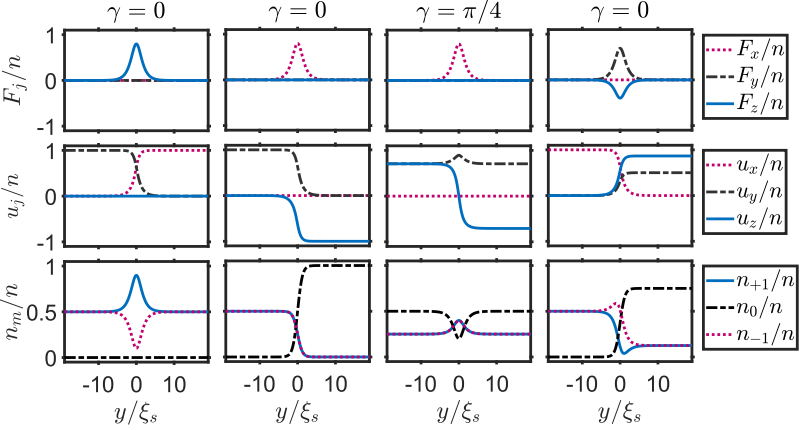

FIG. 1. (a) and (b) Spin density field $\boldsymbol{F}$ and director field $\boldsymbol{u}$ of (a) a magnetic soliton and (b) a domain wall magnetic soliton (DWMS), as described in the text. The blue and red arrows are the spin density and the director, respectively. The blue curves are the magnetization profile. (c) A vector representation of an $\mathrm{SO}(3)$ soliton. The blue and red arrows are the normalized spin density $\boldsymbol{F} / n_{\text {tot }}$ and director $\boldsymbol{u} / n_{\text {tot }}$ evaluated at the center of the soliton, respectively. The blue disk denotes the $x-y$ plane. The red disk denotes the plane normal to the spin vector, on which the director lies. $\alpha, \beta, \gamma$ are Euler angles of the $\mathrm{SO}(3)$ soliton. The additional angle $\pi / 4$ of the red arrow comes from the angle between the $x$ axis and the director before the rotation. (d)-(g) Examples of $\mathrm{SO}(3)$ solitons with different Euler angles. In all cases the soliton velocity is $U=0.6$. The top and middle rows show the spin density and the director, respectively. The red dotted, black dashed, and blue solid lines are vector components in $x, y, z$, respectively. The bottom row shows the density distributions of the three components, where the red dotted, black dashed, and blue solid lines are density distributions of $m=-1,0,+1$, respectively.

freedom, we can construct a new family of solutions to Eq. (1) from $\psi_{\mathrm{MS}}$, namely, the $S O(3)$ soliton solutions,

$$
\psi_{\mathrm{SO}(3)}=\hat{U}(\alpha, \beta, \gamma) \psi_{\mathrm{MS}},
$$

where $\hat{U}(\alpha, \beta, \gamma)$ is the rotation operator acting on the spin degree of freedom and $\alpha, \beta, \gamma$ are the Euler angles. Equation (7) is the principal result of this Letter. We will adopt the $z-y-z$ convention such that $\hat{U}(\alpha, \beta, \gamma)=e^{-i \alpha \hat{F}_{z}} e^{-i \beta \hat{F}_{y}} e^{-i \gamma \hat{F}_{z}}$.

Figure 1(d) shows a plot of all three components of $\boldsymbol{F}$ and $\boldsymbol{u}$, as well as the density profiles of the three spin components $n_{m}, m=-1,0$, and +1 , for an $\mathrm{SO}(3)$ soliton with $\alpha=\beta=\gamma=0$, i.e., a magnetic soliton. Figures 1(b) and 1(e) show a special case of the $\mathrm{SO}(3)$ soliton when the rotation is about the $y$ axis for $\pi / 2$. This solution has its spin aligned with the $x$ direction. It no longer has a density bump (notch) 
in the $m=+1(-1)$ component but, instead, is a domain wall separating two sides of the soliton. The director rotates from the $+y$ direction to the $-z$ direction across the soliton. Correspondingly, on one side of $y=0$ is a pure $m= \pm 1$ state asymptotically, while on the other side it is pure $m=0$. We therefore refer to this particular solution with $x$ polarization as a domain wall magnetic soliton (DWMS).

The $\mathrm{SO}(3)$ soliton solution (7) is parametrized by four free parameters: $\alpha, \beta, \gamma, U$. In Fig. 1(c) we show the spin vector and the director at the center of an $\mathrm{SO}(3)$ soliton. Notice that after an $\mathrm{SO}(3)$ rotation, the spin vector is given by $\boldsymbol{S}=\sqrt{1-U^{2}}(\sin \beta \cos \alpha, \sin \beta \sin \alpha, \cos \beta)^{t}$; that is to say, the first two Euler angles $\alpha, \beta$ control the azimuthal and polar angles of the spin vector of an $\mathrm{SO}(3)$ soliton, as illustrated in Figs. 1(d), 1(e) and 1(g). The soliton amplitude is still related to its velocity as $|\boldsymbol{S}|=\sqrt{1-U^{2}}$. The energy of an $\mathrm{SO}(3)$ soliton is still $n \hbar c_{s}|\boldsymbol{S}|$ thanks to the invariance of energy under $\mathrm{SO}(3)$ rotations. Comparing Figs. 1(e) and 1(f), one can find that the Euler angle $\gamma$ has no effect on the spin but modifies the density distribution and the director of a soliton.

\section{EXPERIMENT}

Here we report experimental evidence for the DWMS, one member of the $\mathrm{SO}(3)$ soliton family. The experiments began with a sodium BEC of $5 \times 10^{6}$ atoms in an elongated dipole trap with oscillation frequencies of $\left(\omega_{x}, \omega_{y}, \omega_{z}\right)=$ $2 \pi \times(380,5.4,380) \mathrm{Hz}$. A bias magnetic field of $72 \pm 4 \mathrm{mG}$ was applied along the $z$ direction which defined the quantization axis. Initially, all atoms were prepared in the $m=0$ state. To generate DWMSs, we first applied an rf pulse along the $y$ direction [see Fig. 2(a)], which effectively rotated the spin of the BEC by $90^{\circ}$, resulting in an $m= \pm 1$ mixture. Subsequently, we cast a magnetic shadow using a far-offresonance laser beam with a top-hat beam profile, as shown schematically in Fig. 2(b). As discussed in [13], each edge of the top hat generated a pair of $\pm z$-oriented $\mathrm{SO}(3)$ solitons propagating in opposite directions. To create the DWMSs, we instead applied another rf rotation about $y$ by $90^{\circ}$ immediately after the magnetic shadow sequence. This realizes DWMSs [SO(3) solitons with $\alpha=0$ and $\beta= \pm \pi / 2$ ], which evolve in the trap for a variable hold time before detection. This method is successful if the system retains spin coherence between the two rf pulses. Experimentally, we have found this to be true for pulse separations up to $200 \mu \mathrm{s}$, limited by ambient magnetic field fluctuations at the milligauss level.

Figures 2(c)-2(e) display Stern-Gerlach images of the condensates for different hold times. Two local relative phase gradients are imprinted in the condensates, as shown in Fig. 2(b). Consequently, we expect four domain walls to be generated, exactly as one sees in the density distributions in the images taken for 15- and 50-ms hold times. The image with the 90 -ms hold time displays a more complicated pattern. We believe this is primarily due to the residual quadratic Zeeman shift of $q=1.43 \mathrm{~Hz}$ present in the experiment. A nonzero value of $q$ has the effect of reducing the symmetry of the spinor condensate from $\mathrm{SO}(3)$ to $\mathrm{SO}(2)$ in the spin space [23]. Consequently, the DWMSs are no longer stationary states.

As in our earlier work [13], we compared our results to numerical simulations of the spin-1 Gross-Pitaevskii equa- (a)

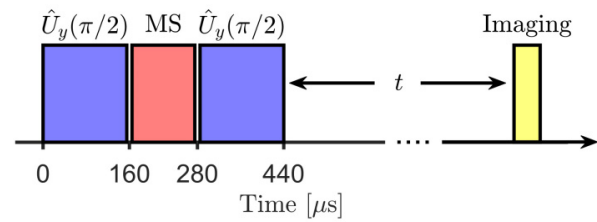

(c)

(d)
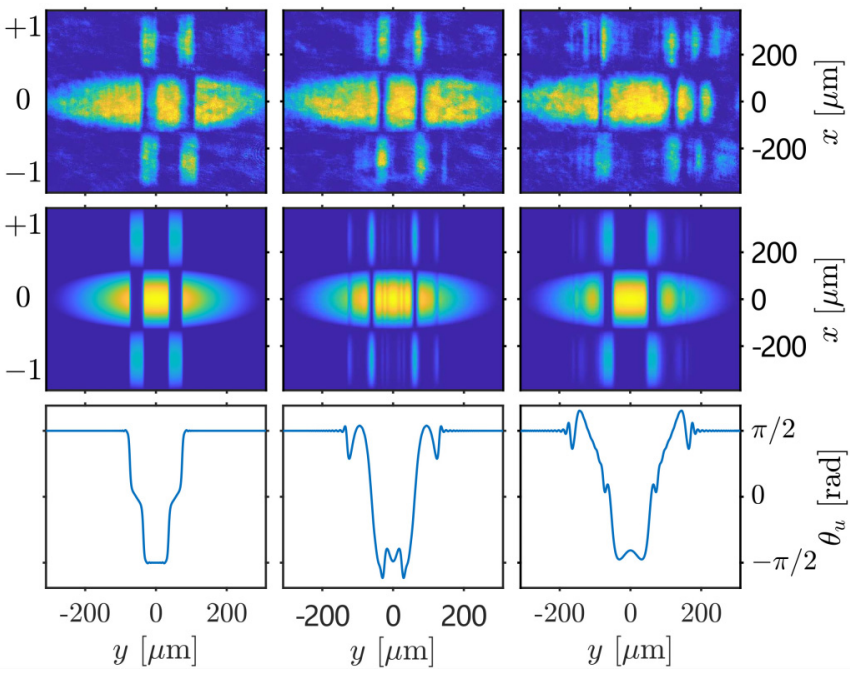

FIG. 2. (a) Experimental sequence for generation of DWMSs in spin-1 BECs. Initially, the condensate is prepared in the $m=0$ state. $\hat{U}_{y}(\pi / 2)$ represents a spin rotation about $y$ for $\pi / 2$. MS means "magnetic shadow." (b) Magnetic shadow. A circularly polarized and properly detuned laser beam illuminates the $m= \pm 1$ BEC along the $z$ direction for a short duration. The pulse induces different Larmor precession rates of the two components, leading to local relative phase gradients. The laser beam is masked by two knife edges, resulting in a magnetic shadow on the condensate. (c)-(e) Evolution of DWMSs in a harmonic trap. The evolution times are (c) $15 \mathrm{~ms}$, (d) $50 \mathrm{~ms}$, and (e) $90 \mathrm{~ms}$. The top panel shows Stern-Gerlach timeof-flight images of the condensates after the generation of DWMSs. The middle panel shows corresponding 1D simulation results with $q=1.43 \mathrm{~Hz}$, where the time-of-flight effects are added manually for visual aid. The simulation time is slightly different from the experimental hold time for reasons discussed in the text. The bottom panel shows the angle $\theta_{u}$ between the director and the $y$ axis. In the simulation the director is always on the $y-z$ plane.

tions. We numerically imprinted a double-edged laser beam on the $m= \pm 1$ BEC and rotated the spin of the BEC by $\pi / 2$ along the $y$ axis with an instantaneous pulse. The system evolved for $100 \mathrm{~ms}$ in the presence of a $q=1.43 \mathrm{~Hz}$ quadratic Zeeman shift, such that $q / g_{2} n_{\text {tot }}(0) \simeq 0.012$. We find from simulation results in Figs. 2(c) and 2(d) that the angle $\theta_{u}$ between the director and $y$ axis shows a $\pi / 2$ rotation across the generated domain wall structures, which is the characteristic feature of an $\mathrm{SO}(3)$ soliton. The angle of the director in Fig. 2(e) shows no sharp jump for the outer two DWMSs, indicating the decay of those two solitons. As shown in the second row of Figs. 2(c)-2(e), the simulation matches the experiments well qualitatively, with residual differences attributed to inhomogeneity of 


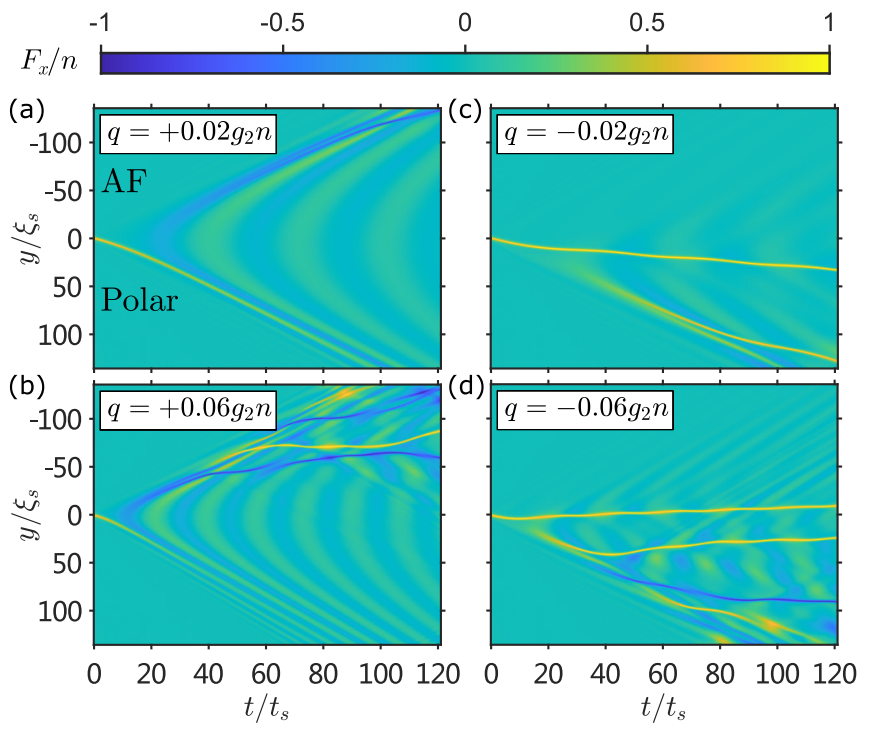

FIG. 3. Space-time plot of $F_{x} / n$ of the $\mathrm{SO}$ (3) soliton by rotating $\pi / 2$ around the $y$ axis with different quadratic Zeeman energies: (a) $q / g_{2} n=0.02$, (b) $q / g_{2} n=0.06$, (c) $q / g_{2} n=-0.02$, and (d) $q / g_{2} n=-0.06$. Here the timescale is $t_{s}=\xi_{s} / c_{s}$. The initial condition is a DWMS given in Fig. 1(e). There are additional magnetic structures in addition to the DWMS appearing in (b) and (d) than in (a) and (c), which implies that the rotated soliton decays faster as the magnitude of quadratic Zeeman energy $|q|$ increases. The emergence of more magnetic structures at $y<0$ (occupied by $m= \pm 1$ atoms) in (b) and at $y>0$ (occupied by $m=0$ atoms) in (d) indicates that (b) the antiferromagnetic and (d) the polar part of the DWMS are unstable.

the phase imprinting beam and its power and imperfect $\mathrm{rf}$ rotation.

\section{STABILITY}

Contrary to our initial expectation that the soliton pair should decay under finite quadratic Zeeman energy $q$, the experimentally observed DWMSs show a quasistable nature. A simple qualitative argument helps us to understand the role of $q$ in the ensuing dynamics. As the DWMSs created by each edge of the laser beam propagate away from each other, they expand the $m= \pm 1$ component. For $q>0$, as in the experiment, this increases the system energy at the expense of soliton kinetic energy. Thus, the quadratic Zeeman shift acts as a "trap" for the solitons. For small $q$ the trap is insufficient to contain the solitons but slows them down, so that they eventually decay into multiple solitonic structures, as shown in the experimental data and the simulations. For large $q$, e.g., $q=0.3 g_{2} n$, by contrast, the stability can be enhanced-we have numerically observed trapping of two DWMSs to form very stable structures.

Our data clearly show that the pairs of DWMSs can be quite stable in the trap, propagating freely for timescales of 50 $\mathrm{ms}$ or so. Their structure at longer times is clearly influenced by the nonzero value of the quadratic Zeeman shift present in the experiment. This is to be expected since the Hamiltonian is no longer $\mathrm{SO}(3)$ invariant. To understand this effect in greater detail we performed numerical simulations of single-soliton (a)

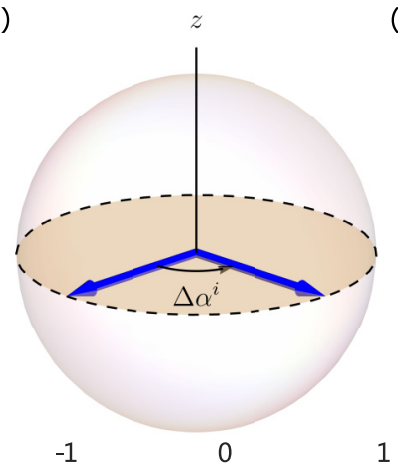

(b)
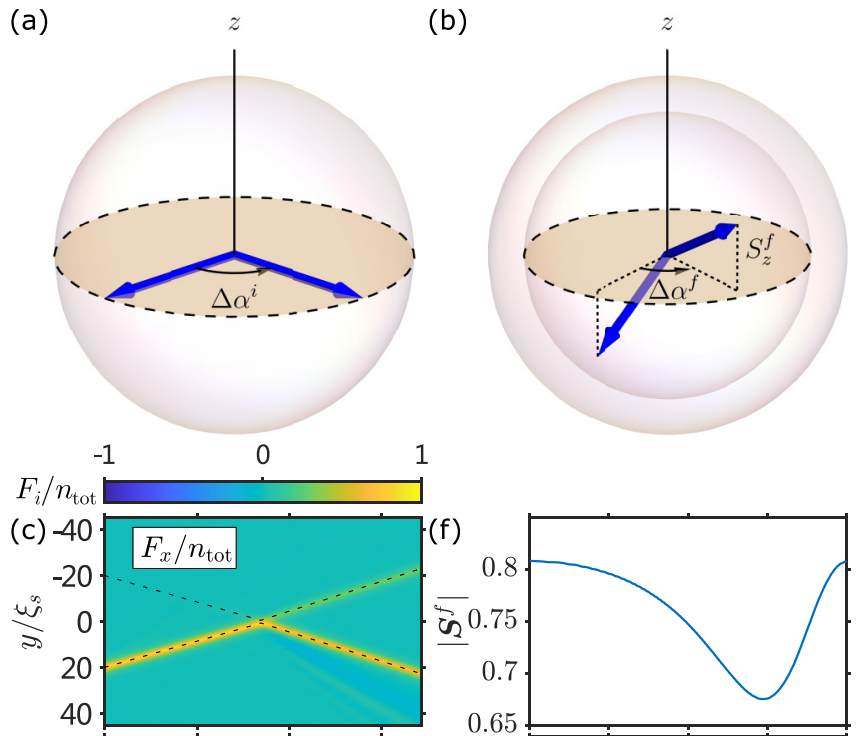

(d) -40

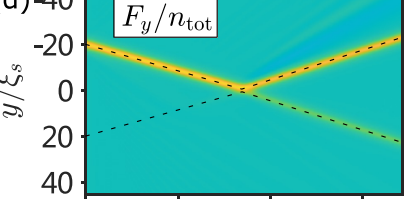

(e) -40

$-40$
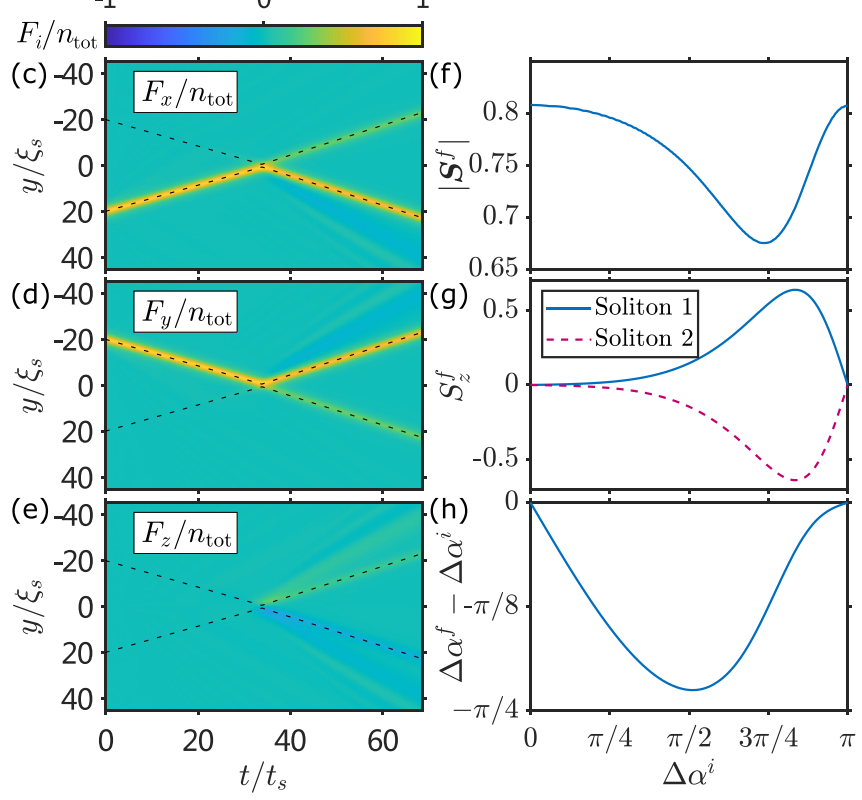

FIG. 4. Collisions of $\mathrm{SO}(3)$ solitons. (a) Initially, the spin vectors, represented as two arrows, are on the $x-y$ plane (the brown disk) with an angle $\Delta \alpha^{i}$ between them. (b) After the collision, the spin vectors of the two solitons precess out of the $x-y$ plane, while both the azimuthal angle difference $\Delta \alpha^{f}$ and the soliton amplitudes $\left|S^{f}\right|$ decrease. (c)-(e) Collisions between two $\mathrm{SO}(3)$ solitons whose parameters are $(\alpha=0, \beta=\pi / 2, \gamma=0, U=-0.6)$ and ( $\alpha=\pi / 2, \beta=\pi / 2, \gamma=0, U=0.6)$, respectively. Plotted are normalized spin densities in the three directions as a function of space and time. Here the timescale is $t_{s}=\xi_{s} / c_{s}$. The dashed lines are trajectories of the two solitons for visual aid. (f) Dependence of soliton amplitudes after collisions on the initial angular difference $\Delta \alpha^{i}$. (g) The blue solid and red dashed lines are $z$ components of the spin vectors of the two solitons after collisions, respectively. (h) Change in $\Delta \alpha$ as a function of $\Delta \alpha^{i}$.

dynamics. They highlight the role of spin-exchange collisions on the soliton stability. Figures 3(a)-3(d) show the magnetization profile of a single DWMS evolving with different $q$. The initial state is a single magnetic soliton in a homogeneous BEC with coherent superposition of $m= \pm 1$ that has been rotated by $\pi / 2$ along the $y$ axis. Multiple magnetic structures show up during the evolution of the soliton, indicating the soliton is unstable and decaying. As the magnitude of quadratic Zeeman energy $|q|$ increases, an increasing number of magnetic structures appear, and the DWMS becomes more unstable. The quadratic Zeeman energy is set up to 
$|q| / g_{2} n=1$ in our simulation; the dynamics of the DWMS is similar to the one in the small- $q$ regime shown in the Fig. 3 . The density profile of this DWMS is shown in Fig. 1(e), which can be naively understood as the superposition of a polar part ( $m=0$ atoms) occupying the $y>0$ region and an antiferromagnetic part ( $m= \pm 1$ atoms) occupying the $y<0$ region, with a small overlap around $y=0$. When $q>0$, the magnetic structures emerge mainly in the $y<0$ region of the condensate occupied by the $m= \pm 1$ atoms since the spinexchange collisions $|0\rangle+|0\rangle \rightarrow|+1\rangle+|-1\rangle$ in the polar part are suppressed by the energy barrier $q$. However, in the $q<0$ regime, more magnetic structures are populated in the $m=0$ region $(y>0)$ since the spin-exchange collisions are energetically favored in the polar part of the soliton.

\section{COLLISION}

Finally, we return to the study of soliton collisions at $q=0$, where striking properties emerge. We have observed that the collisions, while only slightly elastic in nature, cause a nontrivial rotation of the direction of the spin vectors. We numerically examine these collisions in a uniform system. Initially, two $\mathrm{SO}(3)$ solitons are prepared in the condensate, separated by $40 \xi_{s}$ and moving towards each other with $U=$ \pm 0.6 (so that the initial amplitudes of the two solitons are the same, $\left.\left|S^{i}\right|=0.8\right)$. The spin vectors of the two solitons are on the $x-y$ plane with an angle $\Delta \alpha^{i}$ between them, as illustrated in Fig. 4(a). After the collision, as shown in Fig. 4(b), the spin vectors precess out of the $x-y$ plane and also nod towards each other. As an example, Figs. 4(c)-4(e) show typical collisional dynamics of two $\mathrm{SO}(3)$ solitons with spin vectors initially along $+x$ and $+y$. Spin waves are radiated during the scattering of the solitons, indicating the inelastic nature of such collisions.

We then examine the dependence of $\mathrm{SO}(3)$ soliton collisions on the initial angle $\Delta \alpha^{i}$ between the spin vectors of the two solitons. Figure 4(f) illustrates the amplitudes of the spin vectors after a collision as a function of $\Delta \alpha^{i}$. Since the energy of an $\mathrm{SO}(3)$ soliton is proportional to its spin vector amplitude $|\boldsymbol{S}|$, a decrease in $|\boldsymbol{S}|$ indicates energy loss. Although the collisions with $\Delta \alpha^{i}=0$ or $\pi$ are elastic, in general cases the solitons have energy loss after a collision, and the loss is maximized at a certain angle. We suspect the inelastic nature of $\mathrm{SO}(3)$ soliton collisions is due to the nonintegrability of our system. Figure 4(g) shows the collisions induce a nonzero $z$ component of the spin vectors. Because of the conservation of total spin, the $z$ components are opposite for the two solitons after the collision. The two solitons undergo nutation as well, where the nutation angle is shown in Fig. 4(h). We remark that the polarization shift of $\mathrm{SO}(3)$ solitons induced by collisions is quite similar to that of dark-bright-bright solitons in a threecomponent Manakov system [24,25]. Spin precession is also reported for solitons in an integrable spin-1 system [26]. Such similarities indicate possible universal connections between different types of solitons carrying spin. Unlike our system, the integrability of a Manakov system or an integrable spin- 1 system guarantees the conservation of energy of solitons after collisions.

\section{CONCLUSION}

In summary, we have constructed and experimentally observed signatures of a family of $\mathrm{SO}(3)$ soliton solutions to the three-component spin-1 GPEs for antiferromagnetic interactions. We hope our work will invoke follow-up experimental studies of $\mathrm{SO}(3)$ soliton collisions. Theoretical problems, e.g., the physical interpretation of such collisional behaviors in nonintegrable systems, remain to be explored. The stability of $\mathrm{SO}(3)$ solitons created under controlled conditions in higher spatial dimensions is an intriguing avenue for future exploration. Recently, a soliton phase diagram was investigated in a spin-1 Bose gas [27,28] and includes different types of solitons. It is interesting to explore such solitons for a unified view of understanding spinor nonlinear waves beyond magnetic solitons.

\section{ACKNOWLEDGMENTS}

This work acknowledges NSF Grant No. 1707654, JSPS KAKENHI (Grants No. JP19H 01824, No. JP19K14628, No. 20H01843), the Program for Fostering Researchers for the Next Generation (IAR, Nagoya University), and Building of Consortia for the Development of Human Resources in Science and Technology (MEXT).
[1] S. Burger, K. Bongs, S. Dettmer, W. Ertmer, K. Sengstock, A. Sanpera, G. V. Shlyapnikov, and M. Lewenstein, Dark Solitons in Bose-Einstein Condensates, Phys. Rev. Lett. 83, 5198 (1999).

[2] J. Denschlag, J. E. Simsarian, D. L. Feder, C. W. Clark, L. A. Collins, J. Cubizolles, L. Deng, E. W. Hagley, K. Helmerson, W. P. Reinhardt, S. L. Rolston, B. I. Schneider, and W. D. Phillips, Generating solitons by phase engineering of a BoseEinstein condensate, Science 287, 97 (2000).

[3] L. Khaykovich, F. Schreck, G. Ferrari, T. Bourdel, J. Cubizolles, L. D. Carr, Y. Castin, and C. Salomon, Formation of a matterwave bright soliton, Science 296, 1290 (2002).

[4] K. E. Strecker, G. B. Partridge, A. G. Truscott, and R. G. Hulet, Formation and propagation of matter-wave soliton trains, Nature (London) 417, 150 (2002).
[5] C. Becker, S. Stellmer, P. Soltan-Panahi, S. Dörscher, M. Baumert, E.-M. Richter, J. Kronjäger, K. Bongs, and K. Sengstock, Oscillations and interactions of dark and darkbright solitons in Bose-Einstein condensates, Nat. Phys. 4, 496 (2008).

[6] T. Busch and J. R. Anglin, Dark-Bright Solitons in Inhomogeneous Bose-Einstein Condensates, Phys. Rev. Lett. 87, 010401 (2001).

[7] T. M. Bersano, V. Gokhroo, M. A. Khamehchi, J. D’Ambroise, D. J. Frantzeskakis, P. Engels, and P. G. Kevrekidis, ThreeComponent Soliton States in Spinor $F=1$ Bose-Einstein Condensates, Phys. Rev. Lett. 120, 063202 (2018).

[8] H. E. Nistazakis, D. J. Frantzeskakis, P. G. Kevrekidis, B. A. Malomed, and R. Carretero-Gonzalez, Bright-dark soliton 
complexes in spinor Bose-Einstein condensates, Phys. Rev. A 77, 033612 (2008).

[9] Q.-H. Park and H. J. Shin, Systematic construction of multicomponent optical solitons, Phys. Rev. E 61, 3093 (2000).

[10] M. A. Hoefer, J. J. Chang, C. Hamner, and P. Engels, Dark-dark solitons and modulational instability in miscible two-component Bose-Einstein condensates, Phys. Rev. A 84, 041605(R) (2011).

[11] D. Yan, J. J. Chang, C. Hamner, M. Hoefer, P. G. Kevrekidis, P. Engels, V. Achilleos, D. J. Frantzeskakis, and J. Cuevas, Beating dark-dark solitons in Bose-Einstein condensates, J. Phys. B 45, 115301 (2012).

[12] S. V. Manakov, On the theory of two-dimensional stationary self-focusing of electromagnetic waves, Sov. Phys. JETP 38, 248 (1974).

[13] X. Chai, D. Lao, K. Fujimoto, R. Hamazaki, M. Ueda, and C. Raman, Magnetic Solitons in a Spin-1 Bose-Einstein Condensate, Phys. Rev. Lett. 125, 030402 (2020).

[14] A. Farolfi, D. Trypogeorgos, C. Mordini, G. Lamporesi, and G. Ferrari, Observation of Magnetic Solitons in Two-Component Bose-Einstein Condensates, Phys. Rev. Lett. 125, 030401 (2020).

[15] X. Yu and P. B. Blakie, Dark soliton-like magnetic domain walls in a two-dimensional ferromagnetic superfluid, arXiv:2008.08175.

[16] Defined as

$$
\begin{aligned}
& \hat{F}_{x}=\frac{1}{\sqrt{2}}\left[\begin{array}{ccc}
0 & 1 & 0 \\
1 & 0 & 1 \\
0 & 1 & 0
\end{array}\right], \hat{F}_{y}=\frac{i}{\sqrt{2}}\left[\begin{array}{ccc}
0 & -1 & 0 \\
1 & 0 & -1 \\
0 & 1 & 0
\end{array}\right], \\
& \hat{F}_{z}=\left[\begin{array}{ccc}
1 & 0 & 0 \\
0 & 0 & 0 \\
0 & 0 & -1
\end{array}\right] .
\end{aligned}
$$

[17] C. Qu, L. P. Pitaevskii, and S. Stringari, Magnetic Solitons in a Binary Bose-Einstein Condensate, Phys. Rev. Lett. 116, 160402 (2016).
[18] S. Knoop, T. Schuster, R. Scelle, A. Trautmann, J. Appmeier, M. K. Oberthaler, E. Tiesinga, and E. Tiemann, Feshbach spectroscopy and analysis of the interaction potentials of ultracold sodium, Phys. Rev. A 83, 042704 (2011).

[19] T. Congy, A. Kamchatnov, and N. Pavloff, Dispersive hydrodynamics of nonlinear polarization waves in twocomponent Bose-Einstein condensates, SciPost Phys. 1, 006 (2016).

[20] $\psi_{x}=\left(\psi_{-1}-\psi_{1}\right) / \sqrt{2}, \psi_{y}=-i\left(\psi_{1}+\psi_{-1}\right) / \sqrt{2}, \psi_{z}=\psi_{0}$.

[21] T. Zibold, V. Corre, C. Frapolli, A. Invernizzi, J. Dalibard, and F. Gerbier, Spin-nematic order in antiferromagnetic spinor condensates, Phys. Rev. A 93, 023614 (2016).

[22] L. M. Symes and P. B. Blakie, Nematic ordering dynamics of an antiferromagnetic spin-1 condensate, Phys. Rev. A 96, 013602 (2017).

[23] Y. Kawaguchi and M. Ueda, Spinor Bose-Einstein condensates, Phys. Rep. 520, 253 (2012).

[24] B. Prinari, F. Vitale, and G. Biondini, Dark-bright soliton solutions with nontrivial polarization interactions for the threecomponent defocusing nonlinear Schrödinger equation with nonzero boundary conditions, J. Math. Phys. 56, 071505 (2015).

[25] S. Lannig, C.-M. Schmied, M. Prüfer, P. Kunkel, R. Strohmaier, H. Strobel, T. Gasenzer, P. G. Kevrekidis, and M. K. Oberthaler, Collisions of Three-Component Vector Solitons in BoseEinstein Condensates, Phys. Rev. Lett. 125, 170401 (2020).

[26] J. Ieda, T. Miyakawa, and M. Wadati, Exact Analysis of Soliton Dynamics in Spinor Bose-Einstein Condensates, Phys. Rev. Lett. 93, 194102 (2004).

[27] I.-K. Liu, S.-C. Gou, and H. Takeuchi, Phase diagram of solitons in the polar phase of a spin-1 Bose-Einstein condensate, Phys. Rev. Research 2, 033506 (2020).

[28] G. Katsimiga, S. Mistakidis, P. Schmelcher, and P. G. Kevrekidis, Phase diagram, stability and magnetic properties of nonlinear excitations in spinor Bose-Einstein condensates, arXiv:2008.00475 [New J. Phys. (to be published)]. 